\title{
Neck epidermoid cyst mimicking tuberculosis at ultrasound: Case report and review of literature
}

\author{
Jacopo Cambi ${ }^{1}$, Giovanni Monciatti ${ }^{1}$, Luca Pelliccia ${ }^{2}$, Maria Carla Spinosi ${ }^{1}$, \\ Leopoldo Brindisi ${ }^{1}$, \\ Department of ${ }^{1} E N T$, and ${ }^{2}$ Diagnostic Radiology, University of Siena, Siena, Italy. E-mail: ishajacopo@hotmail.it \\ Received: 6 February 2015, Revised: 28 April 2015, Accepted: 29 July 2015
}

SUMMARY: Cambi J, Monciatti G, Pelliccia L, Spinosi MC, Brindisi L. Neck epidermoid cyst mimicking tuberculosis at ultrasound: Case report and review of literature. Turk J Pediatr 2016; 58: 90-93.

Neck masses in children can be inflammatory, congenital or neoplastic. When a congenital cyst becomes infected repeatedly, it may mimic inflammatory disease and the diagnosis may be challenging with ultrasound. An increasing incidence of infection with non-tuberculous mycobacterial organisms has been observed in recent decades in children with cervical lymphadenopathy. An ultrasound scan performed during the infectious phase of a mass can be misleading and can lead to an incorrect diagnostic hypothesis with the risk of erroneous medical and surgical therapies. We describe a case of a lateral neck epidermoid cyst mimicking and misdiagnosed as a tuberculous lymphadenopathy at ultrasound scan and treated with primary surgical excision. After surgery, a histological diagnosis of an epidermal cyst was made. There were no signs of recurrence during the 12-month follow-up period. A review of the literature and a proposed practice pathway for lateral cervical lymphadenopathy in children is also reported.

Key words: epidermoid cyst, tuberculosis, lymphadenopathy, neck, child, ultrasound.

Neck masses in children can be inflammatory, congenital or neoplastic. Children can be affected by many different infectious and inflammatory processes that manifest at the level of the neck. A careful history and physical examination together with a laboratory and radiological assessment typically allow the otolaryngologist to reduce the diagnostic possibilities significantly.

Congenital neck masses are the second most common type of neck mass in children and consist of developmental anomalies of the muscle, skin, blood vessels, lymphatics and the branchial apparatus. ${ }^{1}$ Epidermoid cysts are the simplest germ cell layer lesion, consisting of only a single layer of ectoderm. They are commonly included in the differential diagnosis of midline neck masses. ${ }^{2}$ Although often associated with an inflamed hair follicle, this cyst does not contain cutaneous adnexal structures, as these are of mesodermal origin. The presence of exposed keratin and/or recurrent inflammation/ infection may produce a local granulomatous reaction. Total surgical excision is required with care taken to completely remove the cyst wall and the enlarging mass, and to minimize spillage of cyst contents to prevent its recurrence. ${ }^{3}$

An increasing incidence of infection with non-tuberculous mycobacterial organisms has been observed in recent decades in children with cervical lymphadenopathy. When these organisms infect the cervical lymph nodes of small children, atypical mycobacteria cause a cervical swelling. In the early stages, differential diagnosis must be made with an underlying lymphoma. If untreated, the skin overlying the lymph node becomes red followed by development of an abscess and fistula. Natural resolution with scarring and puckering of the skin may take some years. ${ }^{4}$

Surgical treatment offers several advantages. Ideally, complete excision of infected lymph nodes at an early stage will both eradicate the disease and confirm the diagnosis. 


\section{Case Report}

A 10-year-old boy living in poor social conditions presented for evaluation of a right neck mass with diameter of about $4 \mathrm{~cm}$ and with intense inflammatory reaction of the surrounding neck tissues. There was no history of trauma or any previous surgery. The mass had been present from about 3 months with a gradually increasing volume but without fever and little pain. Twice, he received antibiotic treatment with ceftriaxone ( $1 \mathrm{~g}$ SID for 8 days) with partial resolution of pain and reduction in mass volume. ENT examination observed a single neck mass at right level IIA; it was palpable, round, and poorly mobile on both deep and superficial planes with the consistency of an inflamed lymph node. No adjacent lymph nodes were found. The skin over the mass showed redness and felt warm to the touch.

Laboratory evaluations are reported in Table I. Chest X-ray was negative for pulmonary tuberculosis but a positive Mantoux test had some influence on the clinical and instrumental evaluation. An ultrasound scan carried out in a period of recurrence of infection documented a "lymphnodal swollen with a diameter of about $35 \mathrm{~mm}$, hypoechoic echotexture and scarcely appreciable hilum. Inflammatory reactive adenopathy around the biggest lymph node". A second pre-operative and postantibiotic ultrasound scan documented an "oval mass of about $35 \times 25 \mathrm{~mm}$ with hypoechoic inhomogeneous echo structure with sporadic hyperechoic spots and intralesional flow signals. Reactive lateral cervical lymphadenopathy" (Fig. 1).

The patient underwent excision of the mass and one neighboring node under general anesthesia. Histopathologic examination revealed a keratinized squamous epithelial lining with the inner surface lined with keratin lamellae with chronic productive inflammation in the cyst and reactive lymphadenitis in the neighboring node. There were no histological findings of malignancy and no microbiological signs of atypical mycobacterium. The fluid purulent content of the mass developed colonies of Staphylococcus epidermidis. Based on these findings, a histological diagnosis of an epidermal cyst was made. There were no signs of recurrence during the 12-month follow-up period.
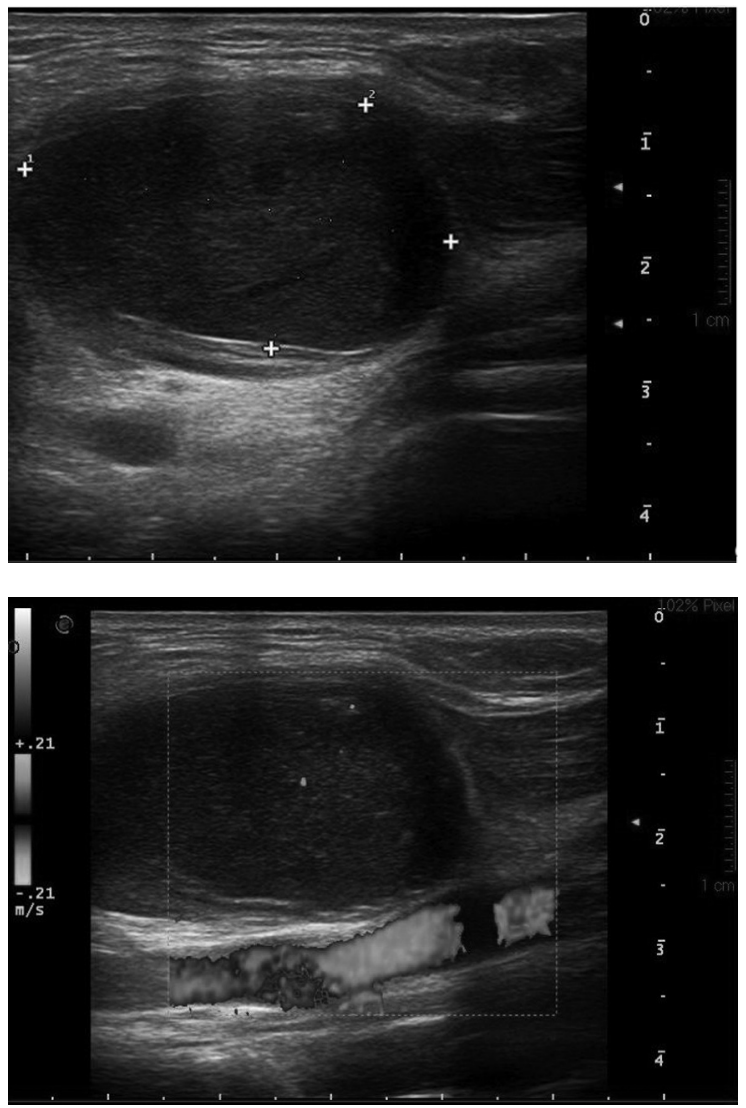

Fig. 1. Upper panel: Ultrasound scan findings after antibiotic therapy: a hypoechoic inhomogeneous round mass with sporadic hyperechoic spots. Lower panel: ColorDoppler interrogation for evaluating vascularity.

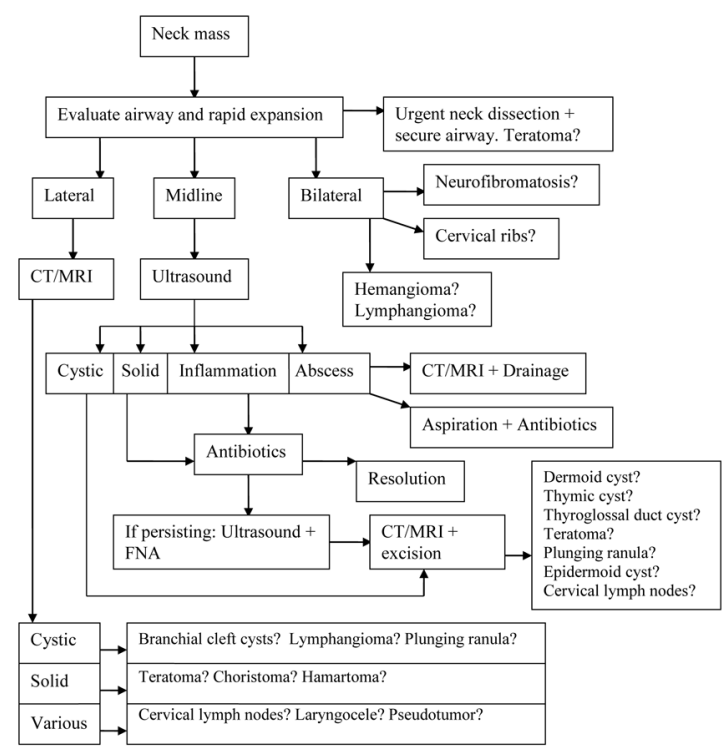

Fig. 2. Algorithm for differential diagnosis, radiology assessment and treatment in pediatric neck masses. 
Table I. Results of Laboratory Tests

\begin{tabular}{lc}
\hline Complete blood count & Leukocytosis $13000 / \mathrm{mm}^{3}$ \\
\hline Monospot testing & Negative \\
EBV & Negative IgM and IgG \\
CMV & Negative IgM; positive IgG \\
Toxoplasmosis & Negative \\
Tuberculin skin test (Mantoux test) & Positive \\
HIV & Negative \\
\hline
\end{tabular}

CMV: cytomegalovirus; EBV: Epstein-Barr virus; HIV: human immunodeficiency virus.

\section{Discussion}

Various theories have been proposed to explain the origin of teratomas (congenital, acquired), but trauma or previous surgery should always be excluded first. ${ }^{5}$ Epidermoid cysts of the neck are quite rare and can reach a large size. Problems such as compression, breathing and/ or swallowing alteration or recurrent infection are described in the literature. ${ }^{6,7}$ Our patient did not report any congenital neck mass or previous surgery.

Epidermoid cysts of the neck grow slowly and do not normally cause symptoms. Most cases are located in the submental region and in the midline of the neck, present as painless superficial masses that move with the skin. They will gradually grow but will rarely become infected. In our patient, the mass was poorly mobile on deep superficial planes as a result of repeated inflammatory episodes following periods of relapse; several inflammatory phases followed one another. When a cyst is infected, radiographic examinations such as ultrasound scans may be misleading. If in fact the epidermoid cyst is a well circumscribed predominantly hypoechoic mass, which if larger, can be a little heterogeneous, during infection, it may be surrounded by soft tissue edema and show a little flow with Doppler examination. These sonographic features are similar to tuberculous lymphadenopathy, which tends to be hypoechoic, rounded in appearance, without an echogenic hilum, and tends to show intranodal cystic necrosis, nodal matting and adjacent soft tissue edema. On Doppler examination, the vascular distribution of tuberculosis nodes is varied and simulates benign and malignant nodes. ${ }^{8}$

On account of the patient's low socio-economic conditions, background and living with foreign nationals, the infectious diseases specialist suspected tuberculosis. This hypothesis was confirmed by a positive Mantoux test, subsequently influencing the radiological diagnosis, moreover, made during a period of acme. These observations make it clear how an ultrasound scan performed during the infectious phase of a mass, as in our patient, can be misleading and can lead to an incorrect diagnostic hypothesis with the risk of erroneous medical and surgical therapies. However, epidermoid cysts should be kept in mind in the differential diagnosis of a cystic neck mass because they are not uncommon in the pediatric population.

The nontuberculous mycobacteria (NTM) are Mycobacterium avium-intracellulare, Mycobacterium scrofulaceum, Mycobacterium bovis and Mycobacterium kansasii. The term scrofula is used nowadays to describe any mycobacterial lymphadenitis. These organisms are ubiquitous in the soil and water, but there is no established human-to-human communication of NTM infection. Generally, it presents as a nontender node that gradually enlarges over several weeks to months in the jugulodigastric or submandibular area. Single node involvement is classic, but synchronous nodal involvement is not uncommon. During surgical excision, our patient's mass was found to be located around the upper third of the internal jugular vein anterior to the vertical plane defined by the spinal accessory nerve lying over the hypoglossal nerve.

For atypical mycobacterium lymphadenopathy in children, the current therapeutic indications are:

Surgical treatment is the therapy of choice. If the lymph node is necrotic or difficult to separate because of branches of the facial 
nerve, only curettage can be made. In the parotid region, a superficial parotidectomy can eradicate early disease. ${ }^{9,10}$

Medical treatment is associated with adverse effects and after several months of treatment, there may be no evidence for a reduction of duration or severity of disease, compared to no treatment, but cosmetic outcome may be improved. Clarithromycin can be used if primary surgical therapy has failed or as a useful adjuvant to surgery. ${ }^{11}$

If lesions are treated non-surgically, then fine needle aspiration (FNA) should be performed to confirm the diagnosis. Unfortunately, this procedure frequently leads to discharge, which may become chronic. ${ }^{12}$ In the pediatric population, this is a procedure to be reserved only for adolescents where FNA can be performed under local anesthesia.

Chest X-ray and Mantoux test should be performed as part of the work-up to exclude tuberculosis. A negative Mantoux test is reassuring, but a positive test is not diagnostic for tuberculosis, as atypical mycobacterial infection may cause false positive results.

Lymphadenitis caused by $M$. tuberculosis is best treated with combination chemotherapy once a proper diagnosis is made. The therapy is similar to that used for pulmonary tuberculosis with combinations of isoniazid, ethambutol, rifampin and streptomycin being the mainstays of therapy. Surgical therapy is rarely necessary.

In the case of lateral cervical lymphadenopathy in children, after history and standard ENT assessment, our current practice pathway is to perform an ultrasound scan to rule out the possibility of an abscess. After any period of inflammation, if present, the ultrasound scan must be repeated to differentiate whether the mass is cystic or solid. If cystic, it can be useful to perform a CT or MRI scan before surgery, especially if the mass is large and has undergone repeated infection. If it is solid and does not respond to antibiotics, it can be useful to perform FNA and if necessary, surgical excision. An algorithm for differential diagnosis and treatment is proposed in Figure 2.

In conclusion, in the reported case, FNA would have excluded atypical tuberculosis with an MRI scan for characterization of soft tissue lesions, and evaluation of features enhanced by injection of contrast medium. We should always aim to reduce the exposure of the pediatric population to the level of radiation produced by CT scans when the disease can be studied with MRI. In a pediatric neck mass, minimizing the risk of radiation and sedation must be considered in the assessment. Recurrent episodes of inflammation/infection and some reported cases of squamous cell carcinoma on epidermoid cysts are strong indications to proceed with surgery.

\section{REFERENCES}

1. Meyer CM. Congenital masses in the neck. In Paparella MM, Shumrick DA, Gluckman JL, et al. (eds). Otolaryngology, Vol. 3. Philadelphia, PA: Saunders; 1991: 2535-2543.

2. Davenport $\mathrm{M} . \mathrm{ABC}$ of general surgery in children. Lumps and swellings of the head and neck. BMJ 1996; 312: 368-371.

3. Erol B, Osma U, Selçuk OT, Et al. Submandibular lateral epidermoid cyst: imaging findings of a rare case. B-ENT 2014; 10: 165-169.

4. Wolinsky E. Mycobacterial lymphadenitis in children: a prospective study of 105 nontuberculous cases with long-term follow-up. Clin Infect Dis 1995; 20: 954-963.

5. Eboriadou M, Al Qassis M, Skouli G, Tzouvelekis G, Hatzibougias D, Athanassiadou F. An unusual presentation of an epidermoid cyst of the neck over the lateral cervical region. J Paediatr Child Health 2008; 44: 601-602.

6. Kang SG, Kim CH, Cho HK, Park MY, Lee YJ, Cho MK. Two cases of giant epidermal cyst occurring in the neck. Ann Dermatol 2011; 23 (Suppl 1): S135-S138.

7. Park TW, Kim JK, Kim JR. Giant epidermal cyst in the posterior neck developing over 40 years: A case report. Exp Ther Med 2014; 7: 287-289.

8. Reynolds JH, Wolinski AP. Sonographic appearance of branchial cysts. Clin Radiol 1993; 48: 109-110.

9. Iversen RH, Illum P. Cervicofacial nontuberculous mycobacterial lymphadenitis in children. Dan Med J 2012; 59: A4349.

10. Lindeboom JA, Kuijper EJ, Bruijnesteijn van Coppenraet ES, Lindeboom R, Prins JM. Surgical excision versus antibiotic treatment for nontuberculous mycobacterial cervicofacial lymphadenitis in children: a multicenter, randomized, controlled trial. Clin Infect Dis 2007; 44 1057-1064.

11. Tessier MH, Amoric JC, Méchinaud F, Dubesset D, Litoux P, Stalder JF. Clarithromycin for atypical mycobacterial lymphadenitis in non-immunocompromised children. Lancet 1994; 344: 1778.

12. Alessi DP, Dudley JP. Atypical mycobacteria-induced cervical adenitis. Treatment by needle aspiration. Arch Otolaryngol Head Neck Surg 1988; 144: 664-666. 